\title{
ENSAYO
}

\section{¿EN QUÉ PARTE DEL ESPECTRO IDEOPOLÍTICO SE PODRÍA UBICAR A SKINNER? UN ANÁLISIS CONCEPTUAL}

\author{
Where in the ideopolitical spectrum could Skinner be located? A \\ conceptual analysis \\ William Montgomery ${ }^{1}$ \\ UNIVERSIDAD NACIONAL MAYOR DE SAN MARCOS \\ PERÚ \\ jmontgomeryu@unmsm.edu.pe \\ https://orcid.org/oooo-0003-2348-5939
}

DOI: https://doi.org/10.35622/j.rr.2022.07.002

Recibido: 10-X-2021 / Aceptado: 16-I-2022 / Actualizado: 10-II-2022

\section{Resumen}

En este artículo se aborda el problema de la posible identificación del principal propugnador del conductismo radical, B. F. Skinner, con alguna ideología política en particular. Para llevar a cabo el análisis se consideraron tres orientaciones ideopolíticas y sus respectivas variantes: anarquismo individualista y colectivista, liberalismo clásico y moderno, y socialismo revolucionario y evolucionista; que fueron confrontadas de manera conceptual con las ideas de Skinner sobre la naturaleza humana, la libertad, la igualdad, la democracia, la perspectiva de sociedad, la idea de autoridad y la economía; obteniéndose una síntesis de las coincidencias que señala una influencia predominante en primer lugar del anarquismo colectivista. Complementariamente se utilizaron los reactivos del test de Nolan para ubicar tales ideas en el espectro ideopolítico, que, según el resultado, estarían inclinadas ligeramente hacia la "centro-izquierda". Al final, se reflexiona críticamente sobre este último punto y sus repercusiones posibles frente a la disyuntiva entre la libertad individual y la convivencia colectiva.

Palabras Clave: Conductismo radical, anarquismo, Ideología, política, liberalismo, socialismo.

\footnotetext{
${ }^{1}$ Psicólogo. Doctorando en Psicología. Magister de Investigación en Comunicación Social. Docente Asociado de la Universidad Nacional Mayor de San Marcos (Lima-Perú) en las asignaturas de Teoría y Técnicas de Intervención Cognitivo Conductual y Epistemología de la Psicología.
} 


\section{Abstract}

This article addresses the problem of the possible identification of the main proponent of radical behaviorism, B. F. Skinner, with a particular political ideology. To carry out the analysis, three ideopolitical orientations and their respective variants were considered: individualist and collectivist anarchism, classical and modern liberalism, and revolutionary and evolutionary socialism; that they were confronted in a conceptual way with Skinner's ideas about human nature, freedom, equality, democracy, the perspective of society, the idea of authority and the economy; obtaining a synthesis of the coincidences that indicates a predominant influence in the first place of collectivist anarchism. Complementarily, the reagents of the Nolan test were used to locate such ideas in the ideopolitical spectrum, which according to the result, would be inclined towards the "center-left". In the end, he critically reflects on this last point and its possible repercussions in the face of the dilemma between individual freedom and collective coexistence.

Keyword: Radical behaviorism, anarchism, Ideology, politics, liberalism, socialism.

\section{DESARROLLO}

El pensamiento del Psicólogo B. F. Skinner (y, por consiguiente, de la corriente conductista radical que impulsó) sigue siendo objeto de controversias respecto a la índole de sus ideas, en especial relacionadas con los aparentes malentendidos que ellas generaron por diversas razones acerca del papel de la cognición, del determinismo, de lo biológico, del tipo posible de teoría y otros múltiples temas. Sin embargo, un punto crucial del rechazo histórico del conductismo radical por parte de mucha gente se centra en las consideraciones que hizo Skinner sobre la sociedad y la posible gubernamentalidad de aquella, más que nada expresadas en obras como Más Allá de la Libertad y la Dignidad (Skinner, 1982); Walden Dos (Skinner, 1985); y Reflexiones sobre Conductismo y Sociedad (Skinner, 1991).

Para unos, el marco filosófico-político del cual parte este referente de la ciencia del comportamiento se halla esencialmente en el fascismo y el conservatismo (Chomsky, 1984; Foucault, 2007, Baum, 2015); y para otros, en una especie de anarquismo o "libertarismo idílico" de izquierda (Dinsmoor, 1992; Rutherford, 2003). De hecho, han habido también enconadas polémicas entre los propios simpatizantes del análisis de la conducta acerca de si la visión conductista de la naturaleza humana se acomoda mejor a las contingencias proporcionadas por el capitalismo (Rakos, 1988ab, 1989), o por las del socialismo (Ulman, 1989, Dittrich, 2019). Como se ve, pues, en este mar de contraposiciones no es cosa fácil decidir por dónde ubicar a Skinner en el espectro ideopolítico.

Dicho espectro, según suele ser presentado por los especialistas en filosofía política (por ejemplo Kyrnlicka, 1995; Sodaro, 2006; Heywood, 2017), consta de una multiplicidad de colores que, generalmente, no son de igual consideración por todos esos teóricos. De entre ellos, la catalogación más detallada es la de Heywood (2017), quien clasifica las ideologías políticas en diez variedades, algunas con subdivisiones muy notorias. Estas son, en el mismo orden en que las presenta dentro de la organización de su obra: liberalismo, 
conservadurismo, socialismo, anarquismo, nacionalismo, fascismo, feminismo, ideología verde, multiculturalismo e islamismo.

La consideración acerca de la relación mayor, menor o inexistente del conductismo radical con dichas corrientes puede ser graduada a priori, descartando aquellas que no tienen, por el momento, desarrollos que crucen sus objetivos de manera aparente con la ciencia del comportamiento tal y como fue formulada por Skinner. Por esta razón se sugiere que el nacionalismo, el multiculturalismo y el islamismo se dejen de lado. En cuanto al feminismo y la ideología verde, si bien podrían tener puntos de contacto con una ciencia del comportamiento atenta al diseño de culturas, no son cosmovisiones ideopolíticas integrales al mismo nivel que otras, así que tienen demasiados aspectos no confrontables. Respecto al conservatismo y al fascismo, es cierto que han sido motivo de especulaciones por parte de los contradictores más encarnizados del conductismo radical, pero resulta claro que se basan en exageraciones poco convincentes. El propio Skinner no parecía tomar muy en serio tales críticas, como se desprende de sus expresiones en debates académicos sobre sus concepciones acerca de la libertad. Se limitaba a señalar que los procedimientos de control pueden servirle a cualquiera (Reinhold, 1972). Por lo demás, el hecho de que hayan en el muestrario histórico tanto críticas de "humanistas conformistas" como de "humanistas contestatarios", según las denominan Dorna y Méndez (1979, p. 65), contribuye a una anulación mutua de ambas tendencias respecto a la acusación de conservatismo.

Quedan en la baraja tres grandes alternativas: el liberalismo, el socialismo y el anarquismo; pero cada una de ellas, a su vez, se halla poblada de variantes. En un esfuerzo por concentrar dichas orientaciones, el citado Heywood las trata así (tabla1):

Tabla 1. Tres orientaciones ideopolíticas y sus respectivas variantes, según las define Heywood (2017).

\begin{tabular}{|c|c|}
\hline $\begin{array}{c}\text { Filosofía } \\
\text { política y } \\
\text { representantes } \\
\text { destacados }\end{array}$ & Variantes más molares \\
\hline $\begin{array}{l}\text { Liberalismo } \\
\text { - Robert Nozick } \\
\text { (1938-2002) } \\
\text { - John Rawls } \\
\text { (1921-2002) }\end{array}$ & $\begin{array}{l}\text { Clásico y moderno: "El liberalismo clásico se caracteriza por la creencia } \\
\text { en un estado "mínimo", cuya función se limita al mantenimiento del } \\
\text { orden interno y la seguridad personal. El liberalismo moderno, por el } \\
\text { contrario, acepta que el estado debería ayudar a la gente a ayudarse a sí } \\
\text { misma" (p. 45). }\end{array}$ \\
\hline
\end{tabular}

Socialismo

- Karl Marx (1818-1883)

- Eduard

Bernstein (1850-1932)

Anarquismo

- Mijail Bakunin
Revolucionario y evolucionista: “[...] los comunistas o marxistas generalmente han apoyado la revolución y han buscado abolir el capitalismo mediante la creación de una sociedad sin clases basada en la propiedad común de la riqueza. En contraste, los socialistas democráticos o socialdemócratas han abrazado el gradualismo y han apuntado a reformar o "humanizar» el sistema capitalista mediante la reducción de las desigualdades materiales y la abolición de la pobreza" (p.115).

Colectivista e individualista: "Los anarquistas hacen especial hincapié en la igualdad política, entendida como un derecho igualitario y absoluto a la autonomía personal, lo que implica que todas las formas 
(1814-1876) de desigualdad política equivalen a opresión. Los anarco-comunistas

- Murray

Rothbard creen en la igualdad social absoluta lograda mediante la propiedad colectiva de la riqueza productiva" (p. 122). "[El anarquismo] puede

(1926-1995) interpretarse como una forma de «ultraliberalismo», que se asemeja al individualismo liberal extremo, o como una forma de «ultrasocialismo», que se asemeja al colectivismo socialista extremo" (p. 160).

Para efectos del presente análisis, se toman como válidos y suficientes los análisis de la tabla 1, aunque se reconozca que aún es posible hacer más subdivisiones entre las alternativas. Dichas caracterizaciones servirán para cumplir el propósito de esta comunicación, que es determinar de manera tentativa cuáles son las cercanías del pensamiento skinneriano con esas filosofías políticas, en términos de afinidad.

La metodología a utilizar será confrontar de manera conceptual las posturas mencionadas frente a temas cruciales que también son enunciados por Heywood (2017): naturaleza humana, libertad, igualdad, democracia, perspectiva de sociedad, idea de autoridad y economía; y luego ubicar las ideas de Skinner en un lugar del espectro ideopolítico utilizando como referencia el test de Nolan.

\section{DESARROLLO}

\section{TEMAS CRUCIALES DE CONFRONTACIÓN}

\section{Naturaleza Humana}

Queda claro que para el análisis experimental de la conducta (Skinner, 1971, 1982) las contingencias derivadas de las influencias genética y ambiental y sus interrelaciones son las que moldean al ser humano, siendo "el medio ambiente social" (Skinner, 1991, p. 17) el que lo dota de su más complejo repertorio de comportamiento. Las circunstancias insertas en las prácticas culturales imponen diferencias entre las personas a través de contingencias de reforzamiento mantenidas por el grupo. Sin embargo, ello no obsta para que también se consideren diferencias personales basadas en la inteligencia, el temperamento y ciertas aptitudes y habilidades no estrictamente desarrolladas a nivel ontogénico (Skinner, 1985, p. 148). Además, las personas actúan sobre el mundo cambiándolo de tal modo que afecta su propio devenir individual y colectivo. En este sentido, el pensamiento skinneriano es compatible en líneas generales con las concepciones socialista revolucionaria del hombre concreto (Sève, 1972) y liberal clásica de la acción humana (Mises, 2014). Esto se complementa con el aserto de que el conductismo radical en compañía del liberalismo, del anarcoindividualismo -y también podría decirse del evolucionismo-, es coherente con el "utilitarismo consecuencialista" (Kyrnlicka, 1995; pp. 12-14): la certidumbre de que el propósito vital y el acto humano son independientes de entidades metafísicas y de tradiciones morales impuestas, estando estructurados más bien en gracia de sus efectos facilitadores de bienestar. Desde la óptica socialista, en cambio, se objeta la idea de "reducir la naturaleza humana" a la "relación única de la utilidad posible" como algo que solo refleja el ideal de bienestar de la sociedad burguesa (Sève, 1972, pp. 104-105). 


\section{Libertad}

El tema más recurrentemente polémico de los escritos skinnerianos es, indudablemente, el de la libertad. Dado que el control de las contingencias es omnipresente, el hombre no puede ser libre en el contexto de un análisis científico, pues toda causa radica "fuera del individuo" (Skinner, 1971; p. 469). Sin embargo, Skinner -aunque nunca lo enfatizó de manera suficiente para ser entendido- sí acepta el sentimiento de libertad de que goza el individuo cuando escapa del control de contingencias punitivas evidentes o no está sometido a ellas (ver Skinner, 1991, pp. 16 y 20), esto es "libertad negativa" tal como la define el liberalismo clásico (De Paula y Laurenti, 2020); aun cuando algunos de los libertarios más conspicuos (por ejemplo Rand, 2009; Hocutt, 2013) han sido ácidos críticos del conductismo radical justamente en base a la creencia de que Skinner rechaza la libertad por completo.

Lo cierto es que, bien mirada, puede notarse una coincidencia entre la perspectiva conductual y el llamado "orden espontáneo" liberal (Hayek, 2019), derivado de la direccionalidad tentativa con que los individuos actúan en busca de sus propios fines. Esto fue advertido por Nozick (1997), quien señala que el mejor marco para una teoría "de las funciones de utilidad podría establecer cómo las oportunidades, las recompensas y los castigos, y las contingencias de refuerzo, se combinan para configurar las funciones de utilidad y las preferencias" (p. 121), y este marco no sería otro que el del condicionamiento operante, cuya noción de comportamiento es similar al que los liberales clásicos (en especial Mises con su concepto de "acción") denominan intencionado. Esto también se condice con el hecho de que, como lo han puntualizado varios analistas de la conducta (Moxley, 2004; Slife y Yanchar, 1999; Carrara y Zilio, 2020), el tipo de determinismo típico del conductismo radical no sería el mecanicista sino el seleccionista, que sustituye la asociación causa-efecto por la relación funcional, la cual supone un margen de variaciones aleatorias en la emisión de la conducta operante.

\section{Igualdad}

El tipo de igualitarismo al que propende Skinner es moral, no jurídico, apartándose en este sentido de la visión libertaria y acercándose al socialismo evolucionista (no al revolucionario, para el cual no puede haber igualdad entre el explotado y el explotador: Lenin, 1973), y al anarquismo colectivista. En su novela Walden Dos (Skinner, 1985) enfatiza las bondades de la uniformidad de acceso a la educación, el trabajo cooperativo y oportunidades para todos, sea en la vida cotidiana de los miembros de la comunidad como en su crianza. Los niños son congregados según su edad primero en cabinas especiales reguladas con ambientación protectora, y luego en salas-dormitorios colectivos. Conforme crecen, se les asignan funciones de trabajo electivo que se remuneran mediante "créditos" de valor proporcional a la dificultad de las actividades físicas o intelectuales desempeñadas, dando acceso a beneficios diversos, pero no hay circulación de dinero ni propiedad privada que no sea de uso personal. En general todo esto se halla en consonancia más que nada con la mentalidad anarco-colectivista y todo tipo de socialismos utópico-libertarios, según lo hace notar Rutheford (2009), quien ha llevado un trabajo de indagación respecto a cómo se han intentado aplicar (con poco éxito) 
estas pautas igualitarias en las comunidades experimentales reales de Twin Oaks y Los Horcones.

\section{Democracia}

La concepción de democracia en Skinner es, por lo menos, conflictiva. En este sentido, por un lado desdeña la democracia representativa tal como se practica en el occidente; es decir, con derechos de sufragio, límites a la autoridad y libertades jurídicas para sus miembros. Le parece ingenua la creencia en personas cuya "bondad innata" (obviamente los políticos) haría que gobernaran con acierto y sabiduría independientes del conocimiento científico (Skinner, 1975). Su idea de democracia pasa por reservar las tareas de gobierno solo a especialistas nombrados por la comunidad ("planificadores") que, a su vez, estén sometidos a un permanente régimen de autofiscalización de sus prácticas experimentales, con especial atención a sujetarse ellos mismos a las reglas que dictan para los demás, siendo tanto "controladores" como "controlados" por el sistema (Altus, 2009). Hay ciertas coincidencias respecto a esta idea de democracia con el liberalismo clásico, el anarquismo y el socialismo revolucionario. En el primer caso, para los liberales clásicos y anarco-individualistas (por ejemplo Hayek, 2019; Rothbard, 2013), el poder de una democracia solo se liga a principios reguladores que limiten la actuación de todos los miembros de la sociedad, ya que por sí misma no asegura nada, debido a que la representatividad de la mayoría de ciudadanos no constituye garantía de buenas decisiones de gobierno. En cuanto al anarquismo colectivista, es conocida la postura de Bakunin (1990) sobre la vacuidad de la democracia representativa, según él hecha únicamente a fin de someter al pueblo "para la prosperidad de la economía capitalista” (p. 241), siendo lo mejor una participación directa y continua de los miembros de la sociedad. El socialismo revolucionario tampoco confía en la democracia representativa, que según Lenin (1973) no es más que la dictadura de una clase por la otra.

\section{Perspectiva de Sociedad}

La sociedad skinneriana se plasma en la noción de la evolución cultural, muy similar a la evolución natural. En ella, los habitantes son tanto producto de la cultura en que viven como factores de cambio de las contingencias que los enmarcan. Según varíen las contingencias sociales de acuerdo con circunstancias planificadas u ocurrentes al azar, incluyendo posible interacción con otras culturas, las prácticas culturales pueden fortalecerse o debilitarse (Skinner, 1982). Esto se podría entender como una especie de armonía natural que concibe el anarquismo de cualquier color, "basada en la disposición humana natural hacia la cooperación y la sociabilidad" (Heywood, 2017; p. 93). El socialismo evolucionista (por ejemplo Bernstein, 2011) y el liberalismo moderno, concretamente el delineado por Rawls (2006), con su teoría de la justicia, podrían también tener alguna complementareidad.

\section{Idea de Autoridad}

Para Skinner (1971) un gobierno es la mayor fuente de control debido a su poder de castigar positivamente o negativamente. En términos técnicos, lo primero tiene que ver con presentar 
una consecuencia aversiva contingente a una instancia de respuesta que se quiere eliminar -como cuando un agente reduce a un individuo agresivo a base de una llave de judo-, y lo segundo retirar una consecuencia reforzante como sanción por haber actuado de manera infractora o no deseable -como cuando se impone una multa. El control instruccional generalizado que se imparte a través de los planos familiar y escolar, es también ejercicio de autoridad contribuyente a mantener el orden social. El control ético es una derivación igualmente instruccional de pautas que justifiquen un actuar de acuerdo con normas legales de regulación, previniendo fuentes de estimulación social aversiva. De acuerdo con Skinner (1971): "Un gobierno que hace el menor uso posible de su poder de castigar es más susceptible de reforzar nuestra conducta de apoyarlo" (p. 372). Luego, la disposición de contingencias de reforzamiento positivo para permitir que los "controlados [hagan] lo que quieren hacer, y no lo que se les obliga a hacer" (Skinner, 1985; p. 292), debe ser la meta de los planificadores y administradores de una comunidad experimental. Esta finalidad podría asemejarse en parte a la buscada por los anarquistas en general.

\section{Economía}

El sistema económico propuesto en la comunidad Walden Dos (Skinner, 1985), está compuesto de unidades cuantitativas llamadas "créditos de trabajo", a través de las cuales se "pagan" los productos y servicios. Tales créditos, existentes como entradas en un libro de cuentas, se concretan mediante labores asumidas voluntariamente por los miembros de la comunidad. Este sistema, visto desde una perspectiva total, se acerca a la lógica socialista revolucionaria de la planificación centralizada, la propiedad común y la igualdad social; pero, como carece de una expresión estatal, parecería más afín al anarquismo colectivista. No obstante, la declaración de Skinner (1971): "Para un individuo, un objeto «vale» lo que está dispuesto a pagar por él" (p. 416), denota cierto influjo de la teoría subjetiva del valor, que es patrimonio reconocido de la economía liberal. Quedaría por determinar en qué situación real de convivencia (colectivista o individualista) funcionaría mejor un sistema económico basado en el consumo e intercambio libre de las personas sin coacción de por medio.

\section{SÍNTESIS: UN LUGAR EN EL ESPECTRO IDEOPOLÍTICO}

De las confrontaciones precedentes podría sugerirse una síntesis de las coincidencias con las diversas filosofías políticas en cada uno de los rubros tratados. Esto, desde luego, con carácter introductorio y tentativo. La tabla 2 resume las coincidencias enmarcándolas en dos grandes tipos de influencias: una prominente y otra importante.

Tabla 2. Posibles influencias de los enfoques ideopolíticos sobre el conductismo radical en diferentes rubros de consideración.

\begin{tabular}{ccc}
\hline $\begin{array}{c}\text { Enfoque ideopolítico / } \\
\text { Rubros }\end{array}$ & Prominente & Importante \\
\hline
\end{tabular}
- Naturaleza humana
- Liberalismo clásico
- Socialismo revolucionario
- Anarquismo individualista
- Liberalismo moderno 


\begin{tabular}{|c|c|c|}
\hline \multirow[b]{2}{*}{ - Libertad } & \multicolumn{2}{|c|}{ ISSN: $2710-0499$ ISSN-L: $2710-0480$} \\
\hline & $\begin{array}{l}\text { - Liberalismo en general } \\
\text { - Anarquismo individualista }\end{array}$ & $\begin{array}{l}\text { - Socialismo evolucionista } \\
\text { - Anarquismo colectivista }\end{array}$ \\
\hline - Igualdad & $\begin{array}{l}\text { - Socialismo evolucionista } \\
\text { - Anarquismo colectivista }\end{array}$ & - Liberalismo moderno \\
\hline - Democracia & $\begin{array}{l}\text { - Anarquismo en general } \\
\text { - Liberalismo clásico }\end{array}$ & - Socialismo revolucionario \\
\hline - Sociedad & - Anarquismo en general & $\begin{array}{l}\text { - Liberalismo moderno } \\
\text { - Socialismo evolucionista }\end{array}$ \\
\hline - Autoridad & - Anarquismo colectivista & - Anarquismo individualista \\
\hline - Economía & - Anarquismo colectivista & $\begin{array}{l}\text { - Socialismo revolucionario } \\
\text { - Liberalismo en general }\end{array}$ \\
\hline
\end{tabular}

Como se ve, desde una perspectiva global la influencia predominante en primer lugar está en el anarquismo colectivista y en segundo lugar en el individualista. En tercer lugar aparece el liberalismo clásico. El socialismo revolucionario, el liberalismo moderno y el socialismo evolucionista tienen presencias importantes. Lo que se desprende de todo esto es un eclecticismo ideopolítico que, al parecer, fue característico de Skinner como persona, siendo admirador de un anarco-individualista eventual como Thoreau, además de ser el propio teórico de Harvard un pensador con ideas radicales no puestas en práctica y a la vez un cumplido ciudadano-modelo de la democracia liberal (Montgomery, 2021).

Resta indagar si es que lo dicho sirve para ubicar a Skinner en algún lugar definido del espectro ideopolítico. A fin de llevar a cabo esta tarea, obviamente se impone, en primer lugar, decidir cuáles de los enfoques consignados se ligan a tendencias de "derecha", de "centro" o de "izquierda"; y en segundo lugar escoger el tipo de cuadrante cuyos ejes serían los más adecuados para tal ubicación. Una posibilidad es el conocido Gráfico de Nolan (1971), a pesar de que eso es difícil porque en su versión actual en inglés (http://www.nolanchart.com/survey-php) incorpora muchos aspectos de coyuntura política que no estaban en vitrina cuando vivía Skinner, aunque conociendo sus puntos de vista generales sobre los rubros antes revisados podrían arriesgarse algunas respuestas adaptadas a su concepción de comunidad experimental.

De acuerdo con ello, es muy probable que Skinner prefiriera que una sociedad conductualmente dirigida, por lo menos en sus inicios, regulara ciertos discursos socialmente indeseables; que proscribiera absolutamente el uso de armas; que no existieran regulaciones acerca de la vida en pareja; que la política exterior de la comunidad no fuera conflictiva con otras; que los ciudadanos estuvieran convenientemente fichados para su mejor identificación; que fueran factibles reajustes impulsados por los planificadores para apoyar ciertos cambios en la producción de subsistencias y también en el comercio; que se utilizaran recursos generales para subvencionar a los que pasaran por situaciones o estados de salud 
difíciles; que todos tuvieran acceso a sistemas de ayuda social y salud; y que la forma de obtención de recursos para el mantenimiento de las estructuras de la comunidad fuera decidida a discreción por su dirigencia. Dando esas respuestas en dicho test, aparece el siguiente resultado (figura 1), que significaría que el lugar del creador del conductismo radical en el espectro ideopolítico estaría en una "centro-izquierda" más tendiente al "intervencionismo gubernativo" (que también podría traducirse como "regulación colectivista”) que a un anarquismo individualista y libertario:

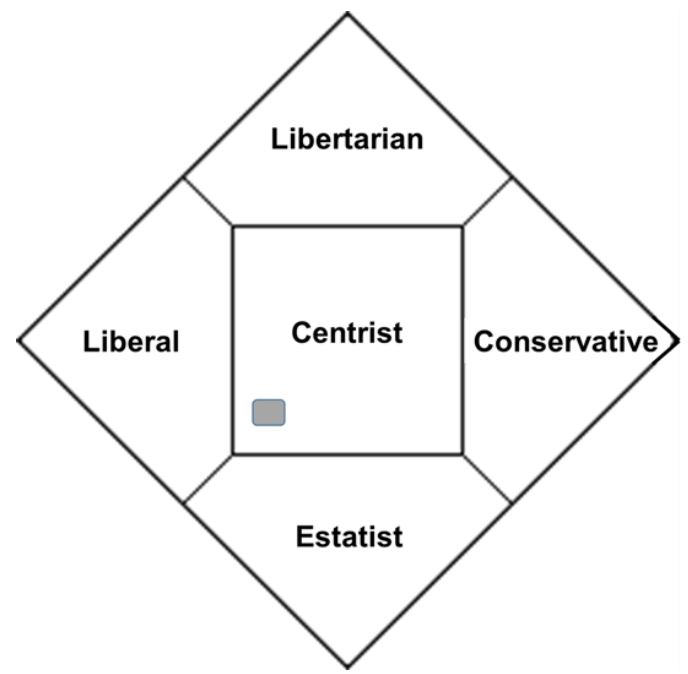

Figura 1. Resultado obtenido en el Test de Nolan, de acuerdo a las respuestas dadas especulando cuáles serían las opciones de B. F. Skinner. Ver: http://www.nolanchart.com/survey-php

De cualquier manera, las consideraciones antes presentadas muestran que Skinner era esencialmente un reformista con influencias diversas.

\section{DISCUSIÓN}

Lo cierto es que el resultado coincide, en líneas generales, con las propuestas del "paternalismo libertario" que provienen del enfoque de economía comportamental de Thaler y Sunstein (2009), según el cual "es legítimo que los arquitectos de las decisiones traten de influir en la conducta de la gente para hacer su vida más larga, más sana y mejor” (p. 19). ¿Cómo?, a través de los "nudges", que en la terminología skinneriana se interpretarían como estímulos discriminativos que hacen más probable la aparición de cierto tipo de respuestas deseables para el individuo y su colectividad, por sobre aquellas otras respuestas que no lo son. De hecho, se puede afirmar que esta estrategia se está aplicando cada vez más en las democracias liberales, donde ya existe una regulación en base, por ejemplo, a símbolos incorporados en las etiquetas de los productos que señalan el nivel de riesgo para la salud que significa consumirlos. $\mathrm{Y}$ en el transcurso de la presente pandemia son ya de presencia cotidiana una serie de modificaciones del ambiente que propenden a facilitar la emisión pública de conductas protectoras, como por ejemplo entre otras las señales en el piso para favorecer el aislamiento social cuando hay colas de espera, o los puestos de aseo y desinfección manual en la entrada de lugares públicos. Las potencialidades de dichos 
procedimientos comportamentales están ilustradas por Krpan, Makki, Saleh, Brink y Klauznicer (2020).

Definida la orientación de Skinner se sugiere que si bien existen beneficios con respecto a este tipo de ubicaciones ideopolíticas, es conveniente añadir que también hay peligros. Una vez traspasado el límite de la intervención sociopolítica en la vida de los ciudadanos "por su propio beneficio", no se sabe hasta dónde se puede llegar. Un caso probable de desembocadura podría ser el de la China Comunista. En ese régimen el uso de la tecnología digital está siendo dirigido hacia un control que por el momento se muestra físicamente poco invasivo (independientemente de la característica vigilancia policíaca del sistema), como es el del modelo de "crédito social" donde se clasifican "buenos" y "malos" ciudadanos, en base a su obediencia de las normas civiles y sanitarias, y se otorgan privilegios a los mejor calificados, como se ve en recientes documentales sobre ello (France 24 Español, 2019; DW Documental, 2021). Sin embargo, semejante forma de control, propio del socialismo revolucionario de todas las épocas y lugares, está basada en la recopilación de una información que sí invade la vida privada, y tiende a hacerlo cada vez más en aras de una finalidad burocrática. Hasta cierto punto, podría aventurarse que el colofón lógico de esa progresiva invasión no podría ser otro que el "1984" de Orwell o, en el mejor de los casos, el "Mundo Feliz" de Huxley, dando la razón finalmente a quienes auguraban ese final a la intentona de Skinner (Rogers y Skinner, 1972; Hocutt, 2013).

Por ello, cabe prevenir sesgos que históricamente han demostrado ser poco funcionales al mantenimiento de pautas democráticas y de respeto a los derechos del individuo. De ahí que no sea aconsejable llevar la crítica del sistema democrático liberal hasta puntos de ruptura en los cuales se justifique su reemplazo por dudosas "democracias directas", lideradas por personas o élites partidarias no sujetas al contracontrol de la sociedad organizada, por más "uso de la ciencia de la conducta" que puedan proclamar. Esto es necesario decirlo, porque tradicionalmente ha existido una tendencia de cierto sector de analistas conductuales a solo asociar los defectos de la convivencia interpersonal en medio de "contingencias injustas" al "sistema capitalista", y eso porque se basan en una lógica de orden declaradamente marxista (por ejemplo Holland, 1978; Ulman, 1991; Carrara, 2005), o bien influidas por esta (como en Dittrich, 2019). Resulta tan poco convincente un sistema de contingencias controladas a casi todo nivel por una autoridad burocrática centralizada en un organismo partidario (característica inevitable del socialismo revolucionario), que otro manejado por una supuesta oligocracia "burguesa". La pretensión skinneriana de que los controladores, siendo guiados por "los principios del experimentalismo y la mejora continua" (Alonso-Vega, et al, 2020, p. 268), se someterían a similar control de las reglas de diseño comunitario a las que sujetarían a los demás miembros de la sociedad, es todavía más ingenua que la de esperar que los políticos demócratas se regulen a sí mismos. De nada sirve tampoco acudir a ejemplos de gobierno autogestionario como el adoptado en la comunidad Los Horcones, porque esta clase de conducción solo es posible debido al pequeño número de miembros que la integran; y ni aun así ha dejado de tener problemas muy serios (Rutheford, 2009). Parece que el mejor contracontrol siempre estará afincado en el respeto fundamental ante los derechos civiles individuales básicos, la tolerancia y la búsqueda de consenso entre representaciones 
ciudadanas (Sodaro, 2006). Ello, con todos sus defectos y dificultades, solamente se ha logrado en cierta medida dentro del diseño institucional de las democracias liberales. Non bene pro toto libertas venditur auro.

\section{CONCLUSIONES}

Las posturas predicadas por cada uno de los enfoques ideopolíticos son impulsadas por valores y principios orientados por tres tipos de metas generales: una dedicada a conservar la tradición y el orden, a veces mediante la imposición (lo que se concibe popularmente como "derecha"); otra animada por la modificación progresiva de ciertos aspectos neurálgicos del sistema ("centro"); y otra que propugna cambios rápidos y a veces violentos ("izquierda"). Entonces, se puede hablar de direcciones conservadora, reformista y revolucionaria. A su vez, dentro de esas direcciones hay variantes que enfatizan más, por un lado, la libertad individual y por otro la convivencia colectiva. De acuerdo al análisis aquí realizado, la postura skinneriana en el espectro ideopolítico parece estar claramente entre las reformistas, en una especie de centro con cierta inclinación de izquierda por el componente colectivista que comprende. Dicha connotación, traducida en términos de las corrientes aparentemente más influyentes en el pensamiento de Skinner, se expresa sobre todo en el anarquismo colectivista y luego en el liberalismo en general, con uno que otro aspecto de tinte socialista. El denominador común de esas orientaciones parece ser su garantía de protección civil en un posible escenario de aplicación de los principios conductuales a nivel macro-contingencial.

\section{REFERENCIAS BIBLIOGRÁFICAS}

Alonso-Vega, J., Ávila, I., Núñez de Prado, M. y Pereira, J. (2020). Análisis de la conducta y prácticas culturales. En M. X. Froxán (Coord.). Análisis funcional de la conducta humana. Concepto, metodología y aplicaciones (pp. 239-273). Pirámide.

Altus, D. E. (2009). B. F. Skinner's Utopian Vision: Behind and Beyond Walden Two. Behavior Analysis, 32(2), 319-335. https://doi:10.1007/BF03392195

Bakunin, M. (1990). Escritos de filosofia política 1: Crítica de la sociedad. (Orig. s/f). Alianza Editorial.

Baum, E. (2015). Controlling minds: Guo Renyuan, behavioral psychology, and fascism in Republican China. The Chinese Historical Review, 22(2), 141-159. http://dx.doi.org/10.1179/1547402X15Z.00000000050

Bernstein, E. (2011). El socialismo evolucionista. (Orig. 1898). Comares.

Carrara, K. (2005). Behaviorismo radical. Crítica e metacrítica (2da. Ed.). UNESP.

Carrara, K. \& Zilio, D. (2020). De Mach a Skinner: A ciência como o behaviorista radical a compreende. Acta Comportamentalia, 28(2), 237-255. http://www.revistas.unam.mx/index.php/acom/article/view/75954/67076

Chomsky, N. (1984). El proceso contra B. F. Skinner. En F. W. Matson (Ed.). Conductismo y humanismo (pp.73-95), Orig. 1973). Trillas.

De Paula, W. F. \& Laurenti, C. (2020). Um diálogo entre I. Berlin e B. F. Skinner a respeito do conceito de liberdade. Revista Perspectivas, 11(1), 17-31. https://doi.org/10.18761/PAC.2020.v11.n1.02 
Dinsmoor, J. A. (1992). Setting the record straight: The social views of B. F. Skinner. American Psychologist, 47(11), 1454-1463. https://doi.org/10.1037/0003066X.47.11.1454

Dittrich, A. (2019). James G. Holland: A análise do comportamento como prática política. Psicologia: Teoria e Pesquisa, 35, Article e3526. https://www.scielo.br/j/ptp/a/QBnDHx7GXP8SGkRg5z7Lfgs/?lang=pt\&format=pdf Dorna, A, y Méndez, H. (1979). Ideología y conductismo. Fontanella.

DW Documental (2021). China ¿Estado policial o laboratorio del futuro? https://www.youtube.com/watch?v=ux547wpAodc

Foucault, M. (2007). Nacimiento de la biopolítica. (Orig. 2004). Fondo de Cultura Económica.

France 24 Español (2019). China clasifica a los buenos y malos ciudadanos a través del crédito social. https://www.youtube.com/watch?v=pZugN-3yn M\&t=103s

Hayek, F. A. (2019). Los fundamentos de la libertad (Orig. 1959). Unión Editorial.

Heywood, A. (2017). Political ideologies. An introduction. Sixth edition. Palgrave-Macmillan. https://fliphtml5.com/momww/ngzk/basic

Hocutt, M. (2013). The fruits and fallacies of Fred Skinner on freedom. The Independent Review, 18(2), 263-278. https:// https://www.independent.org/pdf/tir/tir $1802 \quad 05$ hocutt.pdf

Holland, J. G. (1978). Behaviorism: Part of the problem or part of the solution? Journal of Applied Behavior Analysis, 11(1), 163-174. https://doi.org/10.1901/jaba.1978.11-163

Krpan, D., Makki, D., Saleh, N., Brink, S. I. \& Klauznicer, H. V. (2020). When behavioural science can make a difference in times of COVID-19. Behavioural Public Policy, 1-27. https://doi.org/10.1017/bpp.2020.48

Kyrnlicka, W. (1995). Filosofía política contemporánea. Una introducción (Orig. 1990). Ariel.

Lenin, V. I. (1973). La revolución proletaria y el renegado Kautsky. En Obras, tomo IX (19181919). (Orig. 1918). Progreso.

Mises, L. (2014). Teoria e história. Uma interpretação da evolução social e econômica. (Orig. 1957). Instituto Ludwig von Mises.

Montgomery, W. (2021). Montgomery, W. (2021). Conductismo, libertad y liberalismo: Desencuentros históricos y posibilidades de conciliación filosófica, política y económica. Teoría y Práctica: Revista Peruana de Psicología, 3(2), e51,1-9. https://revistateoriaypractica.com/index.php/rtyp/article/view/51/47

Moxley, R. A. (2004). Pragmatic selectionism: The philosophy of behavior analysis. The Behavior Analyst Today, 5(1), 108-125. https://doi.apa.org/fulltext/2014-44745007.html

Nolan, D. (1971). Classifying and analyzing politico-economic systems. The Individualist, January issue.

Nozick, R. (1997). On austrian methodology. In: Socratic puzzles (pp. 110-141). (Orig. 1977). Cambridge.

Rakos, R. (1988a). Capitalism, Socialism, and Behavioral Theory, Behavior Analysis and Social Action, 6, 16-22. https://doi.org/10.1007/BF03449123 
Rakos, R. (1988b). Scientific Analysis or Political Ideology? Behavior Analysis and Social Action, 6(2), 32.

Rakos, R. (1989). Socialism, Behavioral Theory, and the Egalitarian Society, Behavior Analysis and Social Action, 7, 23-29. https://doi.org/10.1007/BF03406104

Rand, A. (2009). El estímulo y la respuesta. En Filosofía ¿Quién la necesita? (pp. 261-293). (Orig, 1972). Grito Sagrado.

Rawls, J. (2006). Teoría de la Justicia. (Orig. 1971). Fondo de Cultura Económica.

Reinhold, R. (April 21, 1972). B. F. Skinner's. Philosophy fascist? Depends on how it's used, he says. The New York Times, p. 41 .

https://www.nytimes.com/1972/04/21/archives/b-f-skinners-philosophy-fascistdepends-on-how-its-used-he-says.html

Rogers, C. \& Skinner, B. F. (1972). Simposio: Algunos problemas que surgen del control de la conducta humana. En R. Ulrich, T. Stachnik \& J. Mabry (Eds.). El control de la conducta humana. (pp. 525-560) (Orig. 1956). Trillas.

Rothbard, M. N. (2013). Hacia una nueva libertad: El manifiesto libertario. (Orig. 1973). Unión Editorial.

Rutherford, A. (2003). B. F. Skinner's technology of behavior in American life: From consumer culture to counterculture. Journal of the History of the Behavioral Sciences, 39(1), 1-23. https://doi.org/10.1002/jhbs.10090

Rutherford, A. (2003). B. F. Skinner's technology of behavior in American life: From consumer culture to counterculture. Journal of the History of the Behavioral Sciences, 39(1), 1-23. https://doi.org/10.1002/jhbs.10090

Sève, L. (1972). Marxismo y teoría de la personalidad. (Orig. 1969). Amorrortú.

Skinner, B. F. (1971). Ciencia y conducta humana. (Orig. 1953). Fontanella.

Skinner, B. F. (1975). Libertad y control del hombre. En Registro acumulativo (pp. 3-21) (Orig. 1955). Fontanella.

Skinner, B. F. (1982). Más allá de la libertad y la dignidad. (Orig. 1971). Fontanella.

Skinner, B. F. (1985). Walden Dos (Orig. 1948). Orbis.

Skinner, B. F. (1991). Reflexiones sobre conductismo y sociedad. (Orig. 1978). Fontanella.

Slife, B. D. \& Yanchar, S. C. (1999). Conceptions of determinism in radical behaviorism: A taxonomy. Behavior and Philosophy, 27(2), 75-96. https://philpapers.org/rec/SLICOD

Sodaro, M. J. (2006). Política y ciencia política: Una introducción. (Orig. 2004). McGrawHill.

Thaler, R. H. \& Sunstein, C. R. (2009). Un pequeño empujón (Nudge). (Orig. 2008). Santillana.

Ulman, J.D. (1989). Beyond the Carrot and the Stick: A Behaviorological Rejoinder to Rakos. Behavior Analysis and Social Action, 7, 30-34. https://doi.org/10.1007/BF03406105

Ulman, J. D. (1991). Toward a Synthesis of Marx and Skinner. Behavior and Social Issues, 1(1), pp. 57-70. https://doi.org/10.5210/bsi.v1i1.189 